\section{Are racehorses becoming faster?}

SIR-“Why aren't horses faster?" asks W. G. Hill in his comment ' on the study by Gaffney and Cunningham ${ }^{2}$ of a genetic trend in thoroughbred horse-racing performance. We suggest here that today's horses are faster and still improving, if only marginally. Figure 1 in the paper by Gaffney and Cunningham shows that between about 1840 and 1980, English thoroughbred horses reduced their winning times for the St Leger, Oaks and Derby races by about 12,20 and 18 seconds, respectively. The resultant per generation improvements in winning times, assuming an average generation interval of 10.1 years $^{3}$, are 0.4 to 0.8 per cent per generation. The Derby was won in 1988 by Kahyasi, whose time of 2:33.84 bettered by 0.06 seconds the record time set in 1987 by Reference Point: a one-year reduction of 0.04 per cent (again, 0.4 per cent per generation). Such improvements are modest when compared with the 1 to 3 per cent gain per year estimated for traits of economic importance in other species of livestock ${ }^{4}$. Nevertheless the horses are running faster.

Several factors might account for the lower apparent gain in response to selection among thoroughbred horses, in comparison with other categories of livestock subjected to breeding regimens. First, compared with factors such as weight gain, carcass composition, milk yield or fleece thickness selected for in pigs, cattle or sheep, race performance in horses involves such diverse components as muscle mass, relative lengths of limb segments, joint strength and stability, and aerobic capacity. Gains in some of these

\title{
An alternative view of the size of solar cycle 22
}

SIR-Several predictions of the size of the present sunspot cycle have been made ${ }^{1-5}$ over the past year. They all suggest that the present sunspot cycle (cycle 22) will be larger than average, possibly of record size (larger than cycle 19 which had a maximum amplitude of 201.3, in terms of the moving average or smoothed sunspot number) or of near-record size (larger than cycles 21 or 18 , the second and third largest cycles, respectively, in the modern era of sunspot observations)

A common thread in the predictions is that they are based on linear fits between the maximum amplitude of the sunspot cycle and the level of geomagnetic activity near cycle onset. Kane', for example, makes use of the minimum value of the aa geomagnetic index near the sunspot minimum; Gonzalez and Schatten use separately minimum values of either the aa index or the Ap index; Thompson ${ }^{+}$uses the number of 'disturbed days per year' (based on the Ap index); and Brown' uses might even be antagonistic to progress in others. Second, improvements in many domesticated breeds have emphasized characteristics very different from those presumably favoured in wild populations; under such circumstances, the initial gains from artificial selection can be quite rapid. In contrast, improvement in the running speed of horses extends a trend that goes back at least 50 million years to the Eocene; it is likely that current gains are occurring along the distant reaches of an asymptotic curve, where continued selection might be expected to produce relatively less net change. Horse breeders can take comfort from the realization that, however slight their gains might seem per generation, on the average they are hundreds of thousands of times more rapid than examples of vertebrate evolution documented from the fossil record ${ }^{5}$. In fact, to a palaeontologist the morphological correlates of the changes in modern horses might even appear punctuational.

\section{Graduate Program in Genetics,}

David A. ECKHARDT

JONATHAN T. ECKHARDT

Department of Computer Science,

The Pennsylvania State University,

University Park,

Pennsylvania 16802,

USA

\author{
Hill, W.G. Nature 332, 678 (1988) \\ 2. Gaffncy. B. \& Cunningham. E.P. Noture 332,722-724 \\ (I988) \\ Mahon. G.A.T. thesis, Dublin Univ. (1980) \\ 4. Smith, C. R\&D Agric, 1, 79-85 (1984).
}

. Gingerich. P.D. Science 222. 159-161 (1983).

\section{Department of Anthropology and}

cycle 22 suggests that it will have a maximum amplitude larger than the mean maximum amplitude $(=116.3$, based on cycles $10-21)$, but one that is not of record size.

Of several bivariate fits, the most reliable for predicting maximum amplitude, $R(\max )$, is one that uses the minimum annual averages of sunspot number, $R(\min )$, and the Ap index, Ap (min), having a correlation coefficient of 0.997 and a standard error of only 3.9. For cycle $22, R(\min )$ occurred in 1986 , having a value of 13.4 , and $A p(\min )$ occurred in 1987 , having a value of 11.0 , implying that $R(\max )$ should be about $144.6 \pm 7.8$ (a 2- $\sigma$ prediction interval).

Based on cycles 17-21, this particular fit has never erred by more than 4.1 units of sunspot number. Providing that cycle 22 is statistically no different from that of cycles 17-21, one infers that cycle 22 should be smaller than both cycles 21 (164.5) and 18 (151.8) and probably about the size of cycle 11 (140.5), making it the fourth or fifth largest sunspot cycle of the modern record. The value 144.6, a smoothed sunspot-number value, is equivalent to 139.4 when expressed as an annual average sunspot number. This latter value is near to just outside the lower limit reported by Brown for the maximum amplitude of cycle $22(175 \pm 35$, in terms of the annual average). Thus, cycle 22 probably will not be an exceptionally large, record-setting sunspot cycle, although it will be above average in size.

Space Science Laboratory, Robert M. Wilson

NASA Marshall Space Flight Center,

Huntsville,

Alabama 35812

USA

\section{Kanc R.P. Sol Phys, $108,415-416(1987)$ \\ Schatten, K.H. \& Sofia. S. Geophys. Res. Lett. 14, 632-635 (1987) \\ 3. Gonzalez. G. \& Schatten. K.H. Sol. Phys. 114. 189-192 (1987). \\ Thompson. R.J. Austr. Phvs. 25. 17-19 (1988). \\ Brown, G.M. Nantre 333, 121-122(1988) \\ Sargent. H.H. III 28th IEEE Vehic. Téch. Conf. 490-496 (IEEE New York 1978) \\ Wilson, R.M. Geophys. Res. Letl. 15, 125-128 (1988). \\ 'Maize' in Somnathpur, an Indian mediaeval temple} large standard error of es implying that the predictions may not be very reliable. In fact, a comparision of observed and expected values of maximum amplitude for modern era sunspot cycles, based on these single-variate fits, shows that they have differed by as much as 44 units of sunspot number.

In contrast to the above predictions are the so-called 'bivariate' fits ${ }^{6,7}$, each based on the combined effects of a geomagnetic index and the level of sunspot number at cycle onset. Such bivariate fits have been shown to be highly correlative $(r \geqslant 0.95)$ and to have a small standard error of estimate $(\leqslant 10)$, implying that predictions of maximum amplitude based on the bivariate fits are inherently more reliable. Application of selected bivariate tits to
SIR-Although Johannessen claims (Nature 332, 587; 1988) that the object in the hands of the goddess in Somnathpur temple represents a maize ear, there is no evidence of maize cultivation at the time of construction of the temple in the thirteenth century AD, either in Karnataka or elsewhere in peninsular India. Maize cultivation in Karnataka commenced in the mid-1960s.

The similarity of the object described by Johannessen to a maize ear was first D. B. Lawrence (Minnesota University) in brought to our attention by Professor 